\title{
SOBRE EL MOVIMIENTO DE UN CONDUCTOR EN UN CAMPO MAGNETICO
}

ARANDA i OLIVERAS, J.

1.B. «Juan de Austria" de Barcelona

SUMMARY

The classical example of a conductor that moves on metal rails linked at one end in the presence of a uniform magnetic field is explained in many Physics books. An implied simplification, view can lead to misleading conclusions, is generally made whenever the problem is studied from the point of wiew of the Lorentz force even in many famous Physics books. The conventional explanation is fully commented on in this article and the phenomenon is analysed from a dynamic and energetic point of wiew.

\section{. INTRODUCCION}

S habitual en los libros de texto presentar el caso de n conductor rectilíneo que se mueve en un campo hagnético uniforme y perpendicular al plano barrido or el conductor en su movimiento a fin de ilustrar la roducción de una f.e.m. inducida. El valor de ésta tuede deducirse a partir de la fuerza de Lorentz y el esultado, cuando el conductor se mueve sobre guías tetálicas paraleias conectadas por un extremo, coinide con el obtenido ai aplicar la iey de Faraday al ciruito determinado por el conductor y las guías.

e da la circunstancia que en muchos de los textos que usan habitualmente, algunos de ellos de reconocido restigio (Feynman 1982), (Lorrain y Corson 1972), Alonso y Finn 1970), (Sears y Zemansky 1966), (Tiper 1977), (Catala 1977), se analiza el problema meante una simplificación implicita relativa al moviiento de los portadores de carga (responsables de la prriente inducida). Cuando esta simplificación se exende al cálculo de la f.e.m. inducida mediante consieraciones energéticas puede conducir al lector a la rónea conclusión de que la fuerza de Lorentz es la de suministra energía a las cargas lo cual es imposie ya que dicha fuerza es siempre perpendicular al momiento de éstas.

bien un análisis exacto del problema se encuentra el conocido texto de Halliday y Resnick (1974) creeos que adolece de poca claridad tanto por las figuras e acompanan al texto como por la introducción de a fuerza normal cuyo origen queda oscuro.

objetivo de esta artículo consiste en realizar una cria de la exposición usual del problema y presentar un rudio detallado del fenómeno desde el punto de vis- ta dinámico y energético. Creemos que puede ser de utilidad para los profesores de cursos universitarios de Física General y quizá también para los profesores de COU y BUP.

\section{CRITICA DE LA EXPOSICIÓN CONVEN- CIONAL DEL PROBLEMA}

La fig. I muestra el ejemplo típico de un conductor moviéndose a velocidad $\nabla$ sobre dos guias metálicas horizontales y paralelas conectadas por un extremo. El sistema está sometido a un campo magnético uniforme $\overline{\mathrm{B}}$ perpendicular al plano determinado por las guías.

Sobre cada una de las cargas del conductor aparece una fuerza magnética de Lorentz. Sea $q$ un portador, esto es, una carga, supuesta positiva en nuestro razonamiento, que puede moverse con relativa facilidad dentro del conductor. La expresion que usualmente se da para la fuerza magnética que actua sobre q es:

$$
\bar{F}=q \bar{v} \times \bar{B}
$$

caya dirección y sentido se muestran en la fig. 1.

En algunos textos, además, de un modo más o menos explícito, se atribuye a dicha fuerza el trabajo necesario para llevar la carga del extremo inferior al superior del conductor (en la figura 1). Esto es, la fuerza de Lorentz aparece como causa de! campo electromotor que determina la f.e.m. inducida. Se llega así al conocido resultado:

$$
E=B 1 Y
$$


En realidad la expresión (1) sólo es correcta en el supuesto de que los portadores se muevan con la misma velocidad $\bar{v}$ que el conjunto del conductor. $Y$ ello sólo es cierto aproximadamente pues los portadores se mueven respecto al conductor con cierta velocidad $\bar{\Xi}$ (si no fuera así no existiria la corriente inducida). Por supuesto que, en los casos de aplicación práctica, $\bar{u} \ll \bar{v}$ y ello hace de (1) una muy buena aproximación de la expresión exacta:

$\overline{\mathrm{F}}=\mathrm{q} \overline{\mathrm{V}} \times \overline{\mathrm{B}}$

Donde $\bar{V} \cdot=\bar{v}+\overline{\mathrm{u}}$ es la velocidad de los portadores respecto al sistema de referencia del laboratorio. La dirección de la fuerza de Lorentz según la expresión (3) se muestra en la fig. 2 y es, naturalmente, distinta de la representada en la fig. 1.

Se plantea ahora la siguiente cuestion: ¿es también (2), deducida de (1), una expresión aproximada?. La respuesta es: no; la expresión (2) es exacta. Ello se debe, como veremos con detalle más adelante, a que la fuerza dada por (1) es en realidad una de las componentes de la fuerza de Lorentz, exactamente dada por (3), y es esta componente la que realiza un trabajo positivo sobre los portadores pudiendo ser considerada, ella sí, como la causa del campo electromotor. Sin embargo cuando se tiene en cuenta el trabajo realizado por la otra componente de la fuerza magnética resulta, como debe ser, que el trabajo total de la fuerza de Lorentz sobre los portadores es nulo.

Ast, pues, si como es habitual, se mantiene implícita ja aproximación contenida en (1) se conduce al alumno a la grave conclusión de que la fuerza de Lorentz realiza trabajo. Si, por el contrario, y como deberia ser, se hace explícita dicha aproximacion, puede llevarse al estudiante a creer que la expresión que da f.e.m. es aproximada

\section{ANALISIS EXACTO Y DETALLADO DEL FENOMENO}

Un análisis detallado del fenómeno que estudiamos exige distinguir entre la situación en que el conductor comienza a moverse y aquella otra en que ya se mueve a velocidad constante (estado estacionario). Por otra parte se gana en claridad si se comienza estudiando el movimiento de un conductor sin la presencia de las guias.

\subsection{Movimiento de un conductor en un campo magnético}

La fig. 3 muestra un conductor al que, en un instante dado, se comunica una velocidad $\bar{v}$. El conductor se mueve perpendicularmente a un campo magnético uniforme $\overline{\mathrm{B}}$. El movimiento tiene lugar, sobre un plano ho-

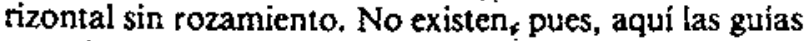
$y$ no hay, por to tanto ningún circuito.

Supongamos que en la situación indicada en la fig. 3 todas las cargas comienzan a moverse a la velocidad $\bar{v}$. Sea $q$ a la carga, supuesta positiva, de un portador.
Sobre él actua tuna fuerza magnética:

$$
\tilde{\mathrm{f}}_{\mathrm{m}}=\mathrm{q} \overline{\mathrm{v}} \times \overline{\mathrm{B}}
$$

La carga $q$ bajo la acción de $\bar{f}_{m}$, y si no existiera interacción con otras cargas, comenzaría a realizar un movimiento circular. Debido a ello aparecen dos compo nentes $V_{x}$ y $V_{y}$ en la velocidad de $q$. Por otra parte el módulo de la velocidad no puede cambiar por ser la fuerza de Lorentz perpendicular al movimiento. Así, pues, $v_{x}<v$. Esto significa que la velocidad de la car. ga $q$ segun el sentido OX se hace inferior a la del conjunto del conductor formado por cargas fijas. En consecuencia dicha carga es «atrapada» por la pared izquierda del conductor. De un modo más preciso: aparece una densidad de carga positiva en la pared opuesta. Dentro del conductor, pues, se crea un campo eléc. trico que llamaremos campo eléctrico transversal $\vec{E}_{t}$ (fig. 4)

Este campo eléctrico realiza sobre un portador $q$ del conductor una fuerza $\bar{f}_{i}=q \bar{E}_{t}$ la cual determina una aceleración de modo que la componente $\mathrm{V}_{\mathrm{X}}$ de dicha carga aumenta. Por supuesto (principio de acción y reaccion) sobre las paredes del conductor aparece una fuerza $\bar{f}_{t}$ que tiende a frenarlo. Ello implica, si se quiere mantener la velocidad $\bar{v}$ constante, la acción de una fuerza externa igual a $\vec{f}_{t}$ sobre el conductor por cada portador que circula en su interior.

La situación estacionaria se conseguirá cuando $\mathrm{V}_{\mathrm{x}}=\mathrm{u}$. Entonces cesará la acumulación de carga en las paredes laterales y la densidad superficial de carga en ellas se mantendrá constante en el tiempo. La fig. 4 muestra las fuerzas que actuan en esta situación sobre un portador cuya velocidad $\bar{V}$ tiene como componentes $V_{x}$ $=v$ y $v_{y}=u$. Observemos la dirección de la fuerza magnética $\bar{f}_{\mathrm{m}}$. Debe cumplirse, obviamente:

$f_{m} \sin \alpha=f_{t}=q B u$

La resultante de las fuerzas que actuan sobre la carga $q$ es, pues, una fuerza dirigida en el sentido oY cuyo módulo es:

$f_{m} \cos \alpha=q B v$

Esta fuerza acelera la carga según $O Y$ de modo que la componente $u$ aumentaría con el tiempo. A ello se opone en primer lugar la interaccion del portador con las cargas fijas del conductor (efecto Joule). Por otra parte el hecho de ser el conductor finito determina una acumulación de carga positiva en su extremo superior y de negativa en el inferior $y$, por tanto, un campo eléctrico longitudinal $\bar{E}_{\text {l }}$ que tiende a frenar el movimiento de la carga $q$ hacia el extremo superior del conductor.

En la fig. $S$ se representan todas las fuerzas que actuan sobre $q$. La fuerza debida al campo eléctrico longitudinal es $\vec{f}_{l}=q \bar{E}_{j} ; \bar{f}_{j}$ puede considerarse como una fuerza de rozamiento que da cuenta del éfecto Joule. A medida que va acumulándose carga en los extremos del conductor va aumentando $\bar{E}_{\text {] }}$. Asi, pues, llega un momento (estado estacionario) en que la componente $u$ 
ie anula con to cual cesa el movimiento de las cargas respecto al conductor. Todas las cargas se mueven ahoa con velocidad $\bar{v}$ por lo que, según (4) la fuerza $\bar{t}_{\text {t }}$ debe ser nula. Ello indica que ha desaparecido la densidad de carga en las paredes del conductor. Su desaparicion se comprende recorriendo a la inversa el proisso que ha conducido a su creación. La nueva situajion dinámica se representa en la fig. 6 .

Obsevemos que, ahora si, la fuerza magnética es perpendicular a la velocidad del conductor y que la fuerla total sobre los portadores del mismo es nula:

$$
\begin{aligned}
\mathrm{f}_{\mathrm{m}} & =\mathrm{f}_{\mathrm{l}} \\
\mathrm{q} \mathrm{B} \mathrm{v} & =\mathrm{q} \mathrm{E}_{\mathrm{l}}
\end{aligned}
$$

Asi, pues, en el estado estacionario, existe un campo eléctrico permanente en el conductor cuya existencia, por otra parte, ha sido confirmada experimentalmenic (Halliday y Resnick 1974)

El hecho de que $\bar{f}_{t}=O$ trae como consecuencia que, en ausencia de rozamiento, no sea ya preciso aplicar tha fuerza externa al conductor a fin de mantener constante su velocidad. Sin embargo, como hemos visto, mientras no se ha llegado al estado estacionario ha estado actuando, por cada portador, una fuerza externa, según (4), igual a:

$$
f_{t}=q B \text { L }
$$

Esta fuerza es la única que puede comunicar energía a las cargas si el conductor debe moverse a velocidad constante. La potencia p correspondiente es:

$$
p=r_{i} v=q B u v
$$

E: trabajo realizado por $\bar{f}_{t}$ hasta liegar at estado esta:innario se invierte en energia interna (efecto joule), nnergia electrostática (campo E) y energía electromagnética (aceleración de las cargas)

\subsection{Movimiento de un conductor sobre guias}

Deseamos ahora estudiar la situación que se produce cuando el conductor anterior, moviéndose estacionariamente, toma contacto con dos guías metálicas paralelas unidas por un extremo.

Como hemos visto existe en el conductor un campo eléctrico $\bar{E}_{1}$ y por tanto una d.d.p. entre sus extremos superior e inferior. Si dichos extremos se ponen en coniacto con las citadas gufas (que cierran un circuito) se producirá una corriente eléctrica, esto es, un movimiento de portadores. Por supuesto ello supone que se rompe el equilibrio en el interior del conductor. Aparece Je nuevo una componente $u$ en la velocidad de los poradores del conductor y se repetirá la situación de la lig. 5. En particulat esto significa que vuelve a actuar tra vez el campo eléctrico transversal $\bar{E}_{t}$ y que se preisa una fuerza externa para mantener la velocidad $\bar{v}$ del conductor. La fig. 7 representa la nueva situación sara un portador $q$ en el conductor. Si la situación es stacionaria debe verificarse:
$f_{\mathrm{t}}=\mathrm{qBu}=\mathrm{f}_{\mathrm{m}} \sin \boldsymbol{\alpha}$

$f_{i}+f_{j}=q B v=f_{m} \cos \alpha$

De todas las fuerzas que actuan sobre $q$ sólo realizan trabajo $\bar{f}_{t}, \bar{f}_{l}, y \bar{f}_{j}$. La primera realiza un trabajo positivo y las otras dos negativo. Esto es, la tinica fuerza que comunica energia a la carga $q$ es $f_{l}$. Naturalmente es ta fuerza externa aplicada al conductor y no dibujada en la fig. 7 la que aporta desde el exterior dicha energia. De otro modo el conductor no mantendria su velocidad constante. La fuerza externa es igual a $\vec{f}_{t}$ por el numero de portadores que se mueven en el conductor.

Desde el punto de vista de las componentes la fuerza que directamente comunica energia a las cargas es, como ya hemos citado en el apartado 2, una de las componentes de la fuerza magnética: $f_{m} \cos \alpha$. El trabajo que esta componente realiza por unidad de carga que se mueve en el conductor es justamente la f.e.m. inducida $E$ cuyo valor es, teniendo en cuenta (7):

$E=\frac{f_{m} \cos \alpha !}{q}=B \vee 1$

Llegamos, pues, al mismo resultado que (2) deducido ahora rigurosamente. Observemos, sin embargo, que la fuerza magnética $f_{m}$ no realiza trabajo. En efecto es inmediato comprobar, teniendo en cuenta su sentido y la expresión (6), que el trabajo correspondiente a la componente $f_{m} \sin \alpha$ es igual y de signo opuesto al de la componente $f_{\text {in }} \cos \alpha$. La única fuente de energia es en realidad, el trabajo de la fuerza externa cuyo valor es, por unidad de carga que se mueve en el conductor:

$$
f_{t} \vee \Delta t / q
$$

donde $\Delta t=V / u$ es el tiempo durante el que actua $f_{t}$ sobre cada carga libre que recorre el circuito. Puede verse, teniendo en cuenta (6), que la expresión anterior coincide con la f.e.m. dada por (8) lo cual confirma que es efectivamente el trabajo de la fuerza externa el origen de la energía asociada a la f.e.m. inducida.

\section{CONCLUSION}

Se ha mostrado cóno el uso implicito de una aproximación en el análisis del movimiento de los portadores en el problema de un conductor que se mueve sobre guias metálicas en presencia de un campo magnético puede conducir a consideraciones erróneas relativas al papel de la fuerza de Lorentz en el balance energético del fenómeno. Y que incluso poniendo de manifiesto la aproximación, lo cual siempre debería hacerse, la explicación convencional puede llevar a dudar de la exactitud de la expresión de la f.e.m. obtenida.

Se ha visto cómo el riguroso análisis del probłema exi$\mathrm{ge}_{\text {, para un conductor bidimensional, la consideracion }}$ de dos campos eléctricos: uno longitudinal y otro transversal. El primero determina una d.d.p. entre los extremos del conductor y el segundo actua sobre los por- 
tadores permitiendo que sigan al conductor en su movimiento de traslación. Este campo transversal se relaciona con el efecto Hail.

Hemos probado que la f.e.m. inducida puede calcularse a partir de una componente de la fuerza de Lorentz y que el trabajo realizado por esta componente es igual al efectuado por la fuerza externa pero que el trabajo total correspondiente a las dos componentes de la fuerza magnética es cero.

Creemos que, si bien es conveniente que los profesores conozcan el análisis detallado del fenómeno tal como aqui se ha presentado su carácter prolijo to hace inadecuado para fines didácticos con alumnos principiantes. Por otra parte hemos puesto de manifiesto los inconvenientes de la exposición convencional. Por todo ello opinamos que, en los primetos cursos es aconsejable deducir la expresion de la f.e.m. inducida a par-

\section{REFERENCIAS BIBLIOGRAFICAS}

\section{.}

ALONSO, M., FINN, E.J., 1970, Fisica (Fondo Educativo Interamericano: E.E.U.U., Vol. il).

CATALÁ, J., 1977, Fisica General (7* edición: Valencia)

FEYNMAN, R.P., 1972, Fisica, (Fondo Educativo Interamericano: E.E.U.U., Vol !I) tir de la ley de Faraday y emplear la fuerza de Lorentz, a lo sumo, para justiffcar cualitativamente la aparición de corriente inducida. El estudio cuantitativo mediante la fuerza de Lorentz debería realizarse en cursos superiores o con alumnos avanzados. En este caso puede optarse, si se desea, por una exposición también cortecta pero menos detallada que la de este artículo partiendo directamente de la situacion estacionaria sobre las guias (fig. 7) y, prescindiendo de su naturaleza elèctrica, presentar la fuerza $\bar{f}_{q}$ como una fuerza normal realizada por las paredes del conductor; éste es el método empleado por Halliday y Resnick en el texto citado en la Introducción

\section{AGRADECIMIENTO}

Agradezco al Dr. F. Senent, de la Universidad de Valencia, su interés y sus múltiples sugerencias y observaciones.
HALLIDAY, D., RESNICK, R., 1974, Fisica (CECSA: México).

LORRAIN, P., CORSON, D.R., 1972, Campos y Ondas Electromagnéticos (Selecciones Cientfficas, Madrid).

TIPLER, P.A., 1977, Fisica (Reverté, Barcelona, Vol. II)

SEARS, F.W., ZEMANSKY, M.W., 1966, Flsica (Aguilar: Madrid). 


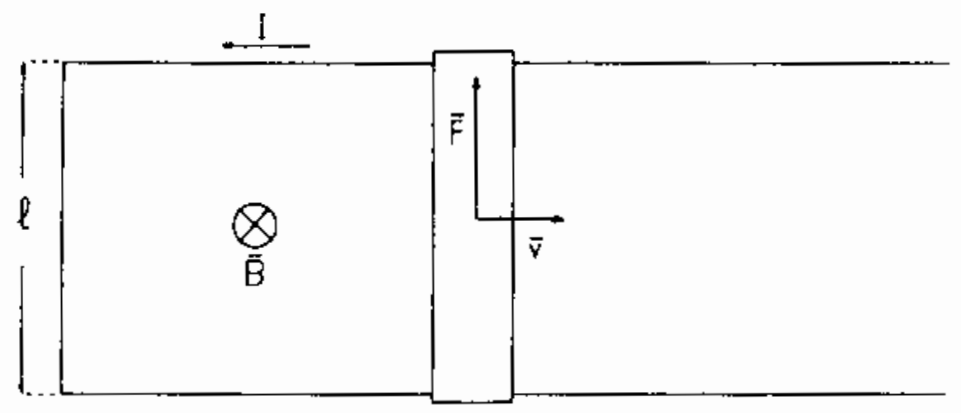

Figura 1

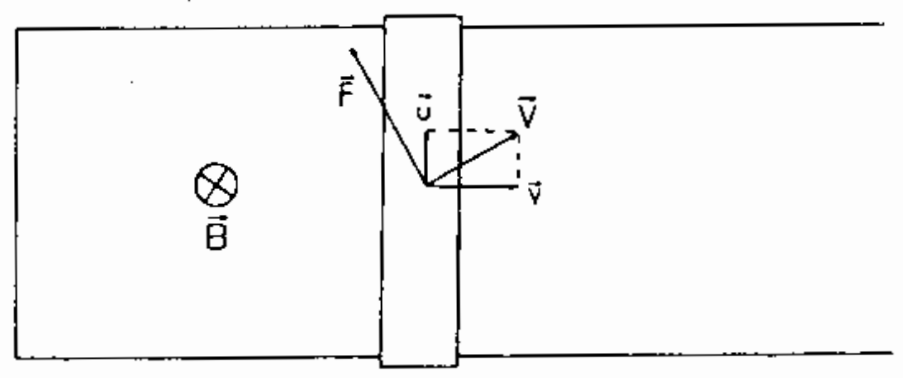

Figura 2

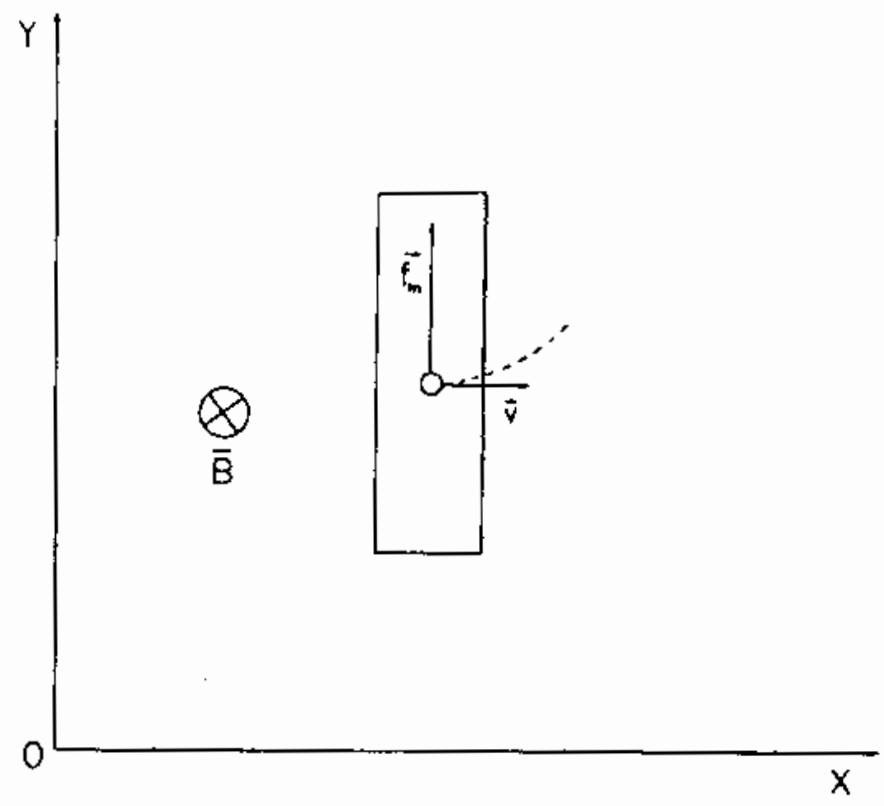

Figura 3 


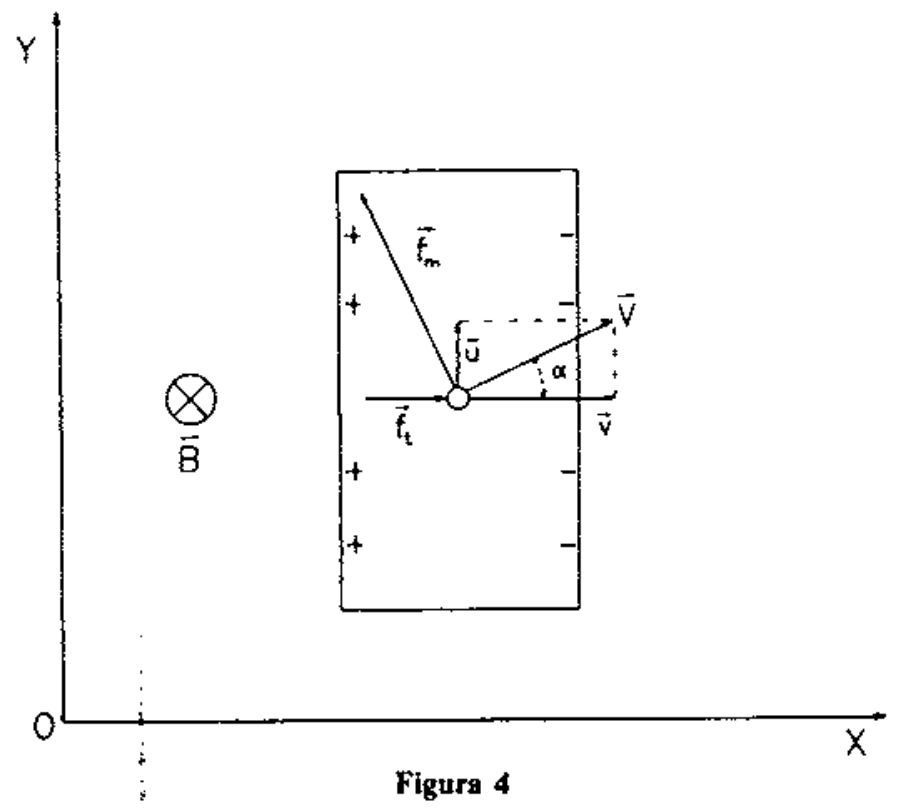

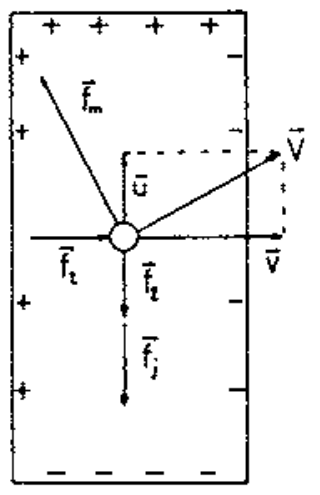

Figura 5

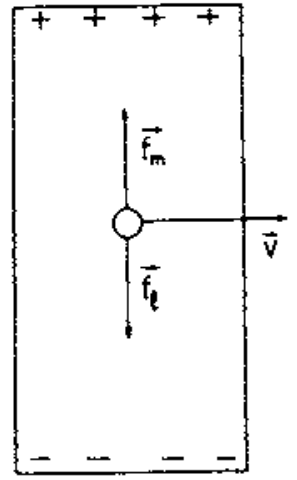

Figura 6

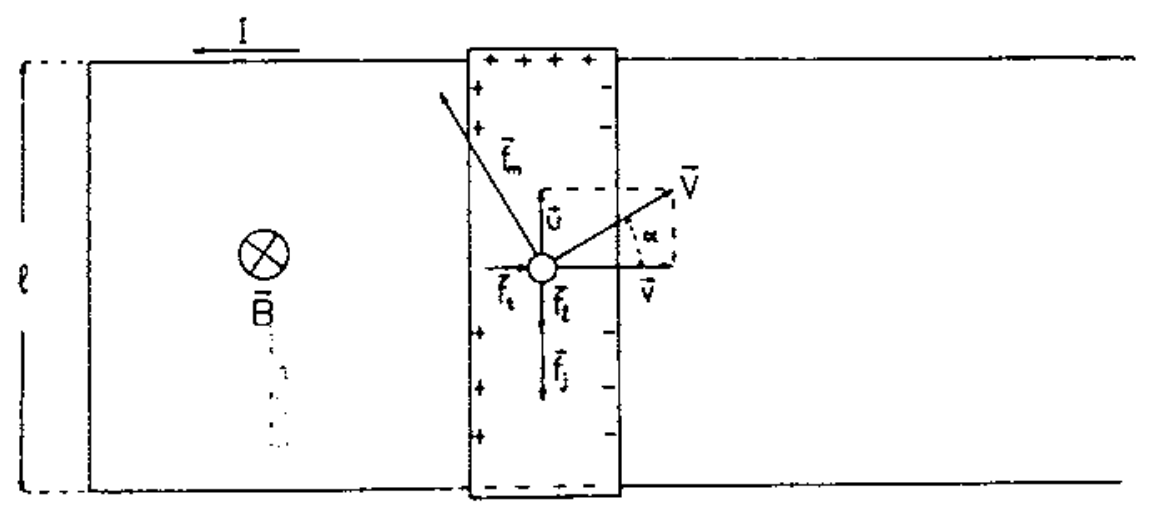

Figura 7 